Meta

Journal des traducteurs

Translators' Journal

\title{
La traduction au magnétophone
}

\section{Lise Laroque-Divirgilio}

Volume 26, numéro 4, décembre 1981

URI : https://id.erudit.org/iderudit/002573ar

DOI : https://doi.org/10.7202/002573ar

Aller au sommaire du numéro

Éditeur(s)

Les Presses de l'Université de Montréal

ISSN

0026-0452 (imprimé)

1492-1421 (numérique)

Découvrir la revue

Citer cette note

Laroque-Divirgilio, L. (1981). La traduction au magnétophone. Meta, 26(4),

398-403. https://doi.org/10.7202/002573ar

Ce document est protégé par la loi sur le droit d'auteur. L'utilisation des services d'Érudit (y compris la reproduction) est assujettie à sa politique d'utilisation que vous pouvez consulter en ligne.

https://apropos.erudit.org/fr/usagers/politique-dutilisation/ 
bureaux (les demi-cloisons) qui ne favorise pas le calme propice à la dictée. On soutient aussi que le traducteur qui écrit son texte peut le modifier à volonté puisqu'il l'a sous les yeux. On semble oublier que celui qui dicte relira aussi son texte tapé et le corrigera au besoin. Il peut aussi très facilement faire marche arrière et réentendre ce qu'il vient de dicter. Il fait alors appel à sa mémoire auditive plutôt que visuelle.

La majorité des traducteurs s'en tiennent peut-être aux méthodes traditionnelles parce qu'ils n'ont pas fourni au magnétophone l'occasion de faire ses preuves; ils ne l'ont pas utilisé suffisamment pour se sentir à l'aise devant les boutons et le micro, et en maitriser le fonctionnement par automatisme. Ce n'est qu'après être parvenu à l'utilisation réflexe de l'appareil que le traducteur pourra concentrer toute son attention sur le texte. Le fil de ses pensées ne sera alors plus constamment interrompu par la transcription de chaque bout de phrase à la main ou à la machine, surtout s'il tape à deux doigts. En effet, la concentration est plus facile lorsqu'il suffit de presser un bouton. «Même s'il est des traducteurs qui se sont habitués à dactylographier directement leurs traductions, cette méthode est difficilement applicable à la traduction de textes complexes. Ceci est dû à l'incompatibilité qui existe entre la traduction, processus mental généralement rapide, et la dactylographie qui requiert du traducteur beaucoup plus d'attention que ne lui en laisse sa concentration mentale sur la traduction. Il s'ensuit nécessairement, soit un rythme ralenti, soit une qualité amoindrie. Et si le traducteur se ravise, il lui sera difficile d'effectuer des changements, par exemple dans l'ordre des éléments d'une phrase [...] l'avantage essentiel de la dictée est que le traducteur ne fait que traduire, l'effort nécessaire pour exprimer oralement sa pensée étant négligeable. De tous les moyens d'exprimer la pensée, la parole, l'écriture ou la dactylographie, la parole est le moyen le plus rapproché de la pensée et qui nécessite le moins d'efforts. Une fois la version définie en esprit et la commande «donnée» pour l'expression orale, la pensée reprend très facilement le fil de la traduction ${ }^{3} »$.

Les adeptes du magnétophone estiment que cette méthode réduit la fatigue et augmente le rendement, mais certains craignent parfois que de leurs traducteurs, n'est-il pas à propos de se demander pourquoi la dictée occupe le dernier rang parmi les méthodes de travail employées, compte tenu du fait que «la méthode la moins productive est celle du brouillon écrit à la main par le traducteur, au fur et à mesure qu'il fait la transposition d'une langue à l'autre ${ }^{2}$ \%.

$\grave{A}$ cet effet. on invoque certaines raisons comme la pénurie de dactylos qui connaissent bien l'orthographe, et l'aménagement des

1. Lise Larocque-DiVirgilio (1980): «Sondage sur les services de traduction dans l'entreprise", Meta, vol. $25, \mathrm{n}^{\circ} 2$

2. Guide du traducteur, ministère des Communications, Service de traduction, Éditeur officiel du Québec, 1978.

3. Guide du traducteur, ministère des Communications, Service de traduction, Éditeur officiel du Québec, 1978. 
la qualité des textes traduits ne souffre au profit de la quantité.

C'est dans le but de savoir si cette impression était fondée que l'auteur, professeur de traduction à l'Université de Montréal, a procédé à l'évaluation méthodique des textes dictés par quarante-trois étudiants de troisième année du baccalauréat, au cours du dernier trimestre de leurs études.

Depuis six ans, la traduction au magnétophone est intégrée à un cours de révision qui se déroule au laboratoire de langues. Il s'agit, pour les étudiants, d'une mise en situation se rapprochant le plus possible du milieu de travail. Au cours d'une séance de trois heures, chacun dicte sa version d'un texte d'environ trois cents mots sur une cassette ordinaire de soixante minutes. L'exercice pourrait aussi se faire avec une cassette de trente ou de quatre-vingt-dix minutes. La consultation des dictionnaires est permise.

Pendant la dictée, le professeur, à la console, passe à l'écoute successive des étudiants, communique avec eux et les conseille jusqu'à ce que l'élocution, le débit et les directives à la secrétaire soient au point.

La dictée terminée, l'étudiant remet sa cassette à un camarade qui en transcrit le contenu à double interligne, de préférence au crayon, pour faciliter les corrections et éviter la multiplicité des ratures. L'exercice de transcription, qui exige une vingtaine de minutes, permet au "Secrétaire" de souligner au traducteur les défauts qui ressortent de sa façon de dicter.

Le texte transcrit est ensuite rendu à son auteur, comme dans un service de traduction. Après avoir corrigé son texte, l'auteur le remet à un camarade qui le révise en rouge.

Chaque étudiant ayant déjà analysé le texte en le traduisant, la révision est accélérée. Il va sans dire que l'interprétation du sens du texte de départ donne parfois lieu à des divergences et à des échanges, comme dans un bureau entre collègues ou entre réviseur et révisé. Au besoin, on fait appel à l'arbitrage du professeur.

À la fin de la séance, les étudiants remettent leurs textes au professeur qui leur attribue une note de traduction et une note de révision. Les corrections du professeur sont en vert, de sorte que le traducteur et le réviseur puissent facilement repérer leurs erreurs la semaine suivante.

Notons qu'à la fin du trimestre, les étudiants terminent leurs traductions et révisions plus tôt qu'au début de la session. Après une dizaine de séances, ils commencent à se sentir à l'aise dans l'emploi de cette méthode de travail. Leurs commentaires les plus fréquents: «Nous aimerions traduire des textes plus longs... Nous aurions dû employer cette méthode plus tôt».
Ces observations, entendues depuis plusieurs années, ne réflétaient toutefois que des impressions. C'est pourquoi nous avons voulu évaluer cette méthode de travail de façon plus rigoureuse.

À la onzième séance, le professeur a donc divisé les étudiants en deux groupes et demandé au premier de traduire un texte au magnétophone et à l'autre de préparer une version manuscrite du même texte. Chaque étudiant devait remplir une fiche de temps indiquant l'heure au début et à la fin du travail pour les textes manuscrits, l'heure au début et à la fin de la dictée, de la transcription et de la correction pour les textes traduits au magnétophone.

La semaine suivante, les rôles furent inversés; ceux qui avaient écrit leur texte la semaine précédente devaient dicter leur version et vice-versa. Chaque semaine, l'ensemble des textes traduits fut remis au professeur qui ne pouvait distinguer les textes manuscrits des textes dictés sans consulter la fiche de temps. Les travaux furent corrigés selon le même barème et le tri effectué après la correction. Les notes obtenues figurent au tableau II.

Nous constatons une économie de vingtquatre minutes, en moyenne, pour traduire 267 mots, ce qui représente une économie de 72 minutes par jour pour 800 mots (rendement quotidien approximatif d'après le sondage de l'auteur auprès des entreprises montréalaises). Sur une semaine de cinq jours, ces 72 minutes quotidiennes représentent six heures, soit une économie de temps de près de $20 \%$, sans diminution de la qualité du travail, car les notes obtenues par les étudiants ayant dicté leurs textes étaient même supérieures de $2 \%$, en moyenne, aux notes obtenues pour les versions manuscrites.

Comme nous le voyons au tableau III, le nombre moyen d'erreurs par étudiant est légèrement moindre pour les versions dictées: premier texte $6,70 / 7,05$; deuxième texte 9,64 / 10,11 .

Il est intéressant de noter, dans ce tableau, que les types d'erreurs sont très semblables, dans les versions dictées et manuscrites, sauf pour les écarts suivants.

Dans les textes manuscrits, on note plus d'erreurs par omission (pertes), soit plus du double pour le texte 1 , et $1,88 / 1,33$ pour le texte 2 .

Les étudiants qui ont écrit leur version ont commis moins d'anglicismes dans le texte 1 $(0,15 / 0.20)$, tandis qu'ils en ont commis beaucoup plus dans le texte $2(0,44 / 0,20)$.

En outre, les erreurs de conjonctions sont plus nombreuses dans la version écrite $(0,15 / 0,10)$ pour le texte 1 et $(0,20 / 0,10)$ pour le texte 2 . 
TABLEAU I

Temps d'exécution

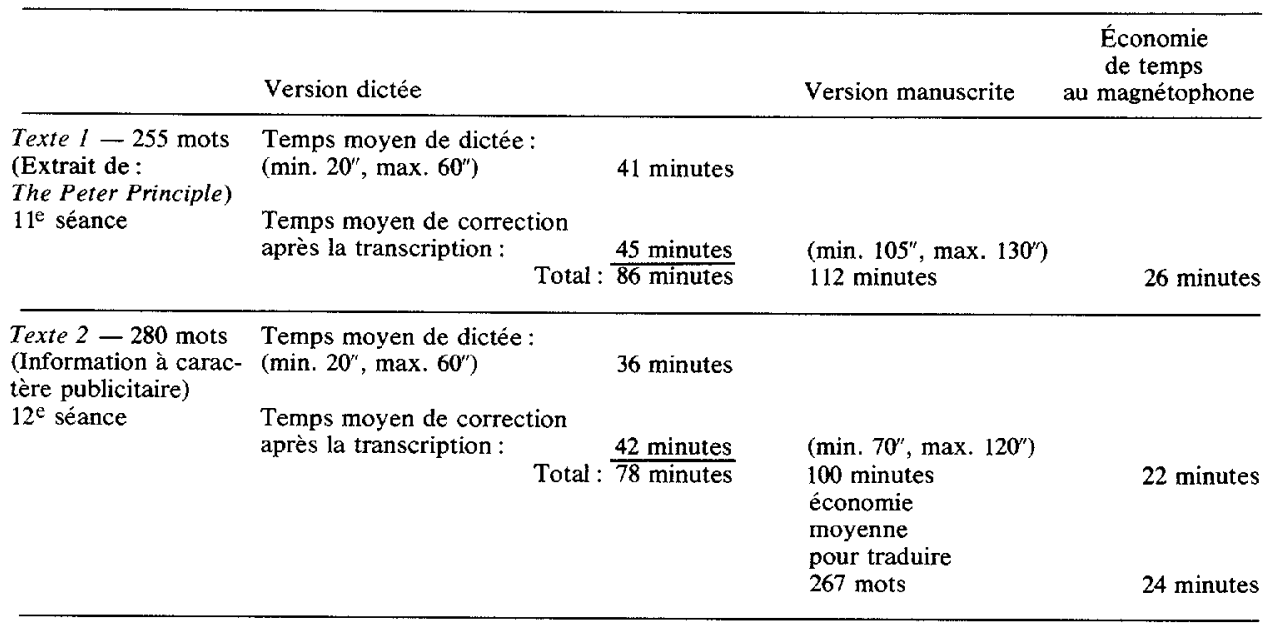

TABLEAU II

Notes obtenues

\begin{tabular}{|c|c|c|c|}
\hline $\begin{array}{l}\text { Texte } 1 \\
\text { Texte } 2\end{array}$ & $\begin{array}{l}\text { Version dictée } \\
76,2 \%(\min .60, \max .82) \\
81,2 \%(\min .52, \max .90)\end{array}$ & $\begin{array}{l}\text { Version manuscrite } \\
71,7 \% \text { (min. } 60, \max .82) \\
81,7 \% \text { (min. } 70, \max .90)\end{array}$ & \\
\hline & $\begin{array}{l}\text { Moyenne des } \\
\text { deux textes } 78,7 \%\end{array}$ & $76,7 \%$ & $\begin{array}{r}2 \% \text { de plus } \\
\text { pour les } \\
\text { versions } \\
\text { dictées }\end{array}$ \\
\hline
\end{tabular}


TABLEAU III

Texte 1

Mauvais choix de substantif

Mauvais choix du verbe

Gains

Pertes

Erreurs dans le temps des verbes

Fautes d'orthographe

Prépositions fautives

Transposition souhaitable

Répétitions

Périphrases au lieu du mot juste

Emploi du passif au lieu de l'actif

Faux sens

Anglicismes

Proverbe mal exprime

Nombre moyen d'erreurs par étudiant

\section{TYPES D'ERREURS}

VERSION DICTÉE VERSIONMANUSCRITE

nombre nombre moyen nombre nombre moyen

total d'erreurs total d'erreurs

d'erreurs par étudiant d'erreurs par étudiants

$\begin{array}{rrrr}31 & 1,55 & 27 & 1,42 \\ 25 & 1,25 & 27 & 1.42 \\ 17 & 0,85 & 12 & 0,63 \\ 6 & 0,30 & 13 & 0,68 \\ 8 & 0,40 & 9 & 0,47 \\ 8 & 0,40 & 6 & 0,32 \\ 8 & 0,40 & 7 & 0,37 \\ 8 & 0,40 & 2 & 0,11 \\ 4 & 0,20 & 8 & 0,42 \\ 2 & 0,10 & 9 & 0,47 \\ 5 & 0,25 & 4 & 0,21 \\ 6 & 0,30 & 3 & 0,16 \\ 4 & 0,20 & 3 & 0,16 \\ 2 & 0,10 & 4 & 0,21 \\ \frac{134}{134} & & 134 & \\ \text { (20 étudiants) } & & & \\ & 6,70 & & 7,05\end{array}$

Texte 2

Mauvais choix du verbe

Pertes

Mauvais choix du substantif

Gains

Périphrases au lieu du mot juste

Faux sens

Fautes d'ortographe

Transposition souhaitable

Répétitions

Prépositions fautives

Anglicismes

Erreurs dans le temps des verbes

Emploi du passif au lieu de l'actif

Charnières mal choisies

\begin{tabular}{rr}
37 & 2,64 \\
19 & 1,36 \\
18 & 1,29 \\
13 & 0,92 \\
11 & 0,79 \\
7 & 0,50 \\
7 & 0,50 \\
6 & 0,43 \\
2 & 0,14 \\
4 & 0,28 \\
3 & 0,22 \\
4 & 0,29 \\
2 & 0,14 \\
2 & 0,14 \\
\hline 135 & \\
(14 étudiants) & \\
& 9,64
\end{tabular}

$\begin{array}{r}18 \\ 17 \\ 9 \\ 9 \\ 6 \\ 9 \\ 9 \\ 3 \\ 5 \\ 2 \\ 4 \\ 0 \\ 0 \\ 0 \\ \hline 91\end{array}$

2,00

1,90

1,00

1,00

0,67

1,00

1,00

0,33

0,55

0,22

0,44

0

0

(9 étudiants) 
Les fautes d'orthographes: 0,40 pour la version dictéc et 0,30 pour la version écrite du texte 1. Par contre, au texte 2 , on note trois fois plus de fautes d'orthographe dans la version manuscrite que dans la version dictée.

Par ailleurs, chez les étudiants qui ont écrit leur texte, la différence est marquée à une rubrique, celle des répétitions, qui sont deux fois plus nombreuses dans le premier texte et cinq fois plus fréquentes dans le deuxième texte.

Dans le premier texte en version dictée, on a omis plus de transpositions $(0,40 / 0.10)$ de même que dans la version $2(0,40 / 0.30)$.

BILAN COMPARATIF DES PRINCIPALES ERREURS

\begin{tabular}{lccc}
\hline & $\begin{array}{c}\text { Version } \\
\text { dictée }\end{array}$ & $\begin{array}{c}\text { Version } \\
\text { manuscrite } \\
\text { moyenne } \\
\text { d'erreurs } \\
\text { par étudiant }\end{array}$ & $\begin{array}{c}\text { Comparaison } \\
\text { moyenne } \\
\text { d'erreurs } \\
\text { par étudiant }\end{array}$ \\
\hline Pertes & 1,66 & 2,58 & $+0,92$ \\
Répétitions & 0,34 & 0,97 & $+0,63$ \\
Anglicismes & 0,42 & 0,60 & $+0,18$ \\
Verbes (choix et temps) & 4,58 & 3,89 & $-0,69$ \\
Faux sens & 0,80 & 1,16 & $+0,36$ \\
Actif/passif & 0,39 & 0,21 & $-0,18$ \\
Transpositions & 0,83 & 0,44 & $-0,39$ \\
Prépositions & 0,68 & 0,59 & $-0,09$ \\
Fautes d'orthographe & 0,90 & 1,32 & $+0,42$ \\
Périphrases & 0,89 & 1,14 & $+0,25$ \\
Total & 11,49 & 12,90 & 1,41 \\
12\% de plus dierreurs en version manuscrite & &
\end{tabular}

Si l'on tient compte du fait que ces étudiants en étaient, pour la plupart, à leur huitième et neuvième versions dictées, (les premières séances du trimestre ayant été consacrées à la théorie ainsi qu'à l'initiation à la dictée et à la révision), il ne fait aucun doute que la plupart des traducteurs en exercice, après quelques semaines de dictée, en arriveraient à un rendement, du moins quantitatif, bien supérieur aux résultats démontrés par la présente évaluation.

D'après un document publié par l'UNESCO${ }^{5}$, «l'enregistrement d'une traduction au dictaphone requiert un sixième du temps nécessaire pour écrire à la main. Même en ajoutant le temps requis pour la transcription de l'enregistrement, la reprise et la correction de la transcription et la nouvelle dactylographie, le temps total représente $50 \%$ du temps requis pour le travail manuscrit. Le coût d'achat du dictaphone se trouve amorti même par une modeste augmentation de productivité du traducteur, vu d'un côté le traitement de celui-ci et de l'autre le coût d'un dictaphone et de la dactylographie ».
En optant pour la dictée, les traducteurs disposeraient de plus de temps pour préparer leurs textes et faire de la recherche terminologique, ce qui devrait améliorer la qualité de leur travail.

Dans la pratique, la dictée représente aussi un avantage appréciable lorsque le traducteur doit exécuter un travail de toute urgence. Il peut alors dicter sur une partie de cassette qu'il remet à la dactylo pendant qu'il poursuit sa dictée sur une autre cassette. Cette technique permet au traducteur et à la dactylo de travailler simultanément, puis au traducteur de commencer à relire son texte tapé dès qu'il en a terminé la dictée.

Certains prétendront que la dictée ne peut être appliquée à tous les genres de textes. Il s'agit là d'une fausse impression, les noninitiés s'imaginant parfois que devant un micro il faille tout dire d'un trait, sans hésiter

5. UNESCO, Scientific and Technical Translating and Other Aspects of the Language Problem. 
ni faire de pause; mais la traduction au magnétophone n'est pas de l'interprétation simultanée. En effet, comme le traducteur qui écrit s'arrête pour chercher un terme, celui qui dicte ferme le micro lorsque le mot juste ne lui vient pas immédiatement à l'esprit. Il peut aussi faire marche arrière et modifier son texte au besoin.

La technique de traduction au magnétophone est la même que pour une version écrite. Il n'est pas nécessaire d'aborder un texte en connaissant à l'avance la solution à tous les problèmes qu'il pose. Après l'avoir lu et compris, s'il n'exige pas de recherche, la méthode la plus rapide consiste à l'aborder et à résoudre les problèmes au fur et à mesure qu'ils se présentent. Devant une difficulté qui semble insurmontable, il est préférable de laisser un blanc, temporairement, plutôt que de s'acharner à résoudre immédiatement l'énigme. Souvent, la solution apparaîtra clairement plus tard au cours de la dictée et de la consultation des dictionnaires qui l'accompagne. S'il subsiste des difficultés non résolues à la fin de la dictée, le traducteur pourra en dresser la liste et effectuer un travail final de recherche muni de tous les éléments du problème. Il lui suffira alors de mettre en place la pièce manquante du puzzle en donnant des directives à la dactylo afin qu'elle remplace un terme ou ajoute le mot juste au bon endroit, ou bien le traducteur s'en chargera à la réception de son texte tapé. Il n'a qu'à noter le bon terme au crayon sur l'original pour ne pas l'oublier.

Un bon nombre d'étudiants passent parfois une heure à consulter les dictionnaires, pour analyser des termes ne présentant pas de difficultés particulières, avant mème d'avoir traduit une seule phrase. Cette recherche fébrile serait un moindre mal si elle améliorait la qualité du travail, mais souvent, plus ils cherchent, plus ils sont indécis devant la multiplicité des choix. Lors des premières séances de dictée, le professeur doit donc les inciter à ranger leurs crayons, sinon certains iront même jusqu'à écrire tout le texte avant de le dicter. Une fois lancés, cependant, ils sont vite «emballés» par les résultats de cette technique, ce qui porte à croire que leurs hésitations viennent de la crainte de l'échec devant des outils mal connus par rapport au bon vieux crayon. L'heure n'est-elle pas venue de songer à délaisser quelque peu ce compagnon traditionnel du traducteur à une époque où la machine à traiter les textes est déjà à l'œuvre dans certains services de traduction?

Lise LARocQue-DiVIRGILIO 